\title{
Ecologie, diversité spécifique et abondance des petits mammifères de la ville de Cotonou au Bénin (Afrique de l'Ouest)
}

\author{
G. HOUEMENOU ${ }^{1 *}$, B. KASSA ${ }^{2}$ et R. LIBOIS ${ }^{3}$ \\ ${ }^{1}$ Laboratoire de Biologie Appliquée, Ecole Polytechnique d'Abomey-Calavi, Université d'Abomey-Calavi, \\ O1BP 2009 Cotonou, Bénin. \\ ${ }^{2}$ Faculté des Sciences Agronomiques, Université d'Abomey-Calavi, O1BP 526 Cotonou, Bénin. \\ ${ }^{3}$ Unité de Recherche en Zoogéographie, Université de Liège, Bâtiment B22 Boulevard du Rectorat, \\ 4031 Sart Tilman, Belgium. \\ *Auteur correspondant, E-mail : gualbert.houemenou@gmail.com
}

\section{RÉSUMÉ}

L'étude de la diversité des petits mammifères de la ville de Cotonou (Bénin) selon les types de milieux (habités ou non) a été réalisée du 25 août 2004 au 25 mai 2005 avec interruptions. Les campagnes de captures ont été effectuées. Au total, 348 petits mammifères ont été capturés pour un effort de piégeage de 2652 nuitspièges et 11 espèces comprenant 3 espèces (Rattus rattus, Rattus norvegicus et Mus domesticus) cosmopolites représentant $25 \%$ du total et les espèces africaines (Crocidura olivieri, Crocidura sp., Arvicanthis niloticus, Cricetomys gambianus, Dasymys rufulus, Mastomys sp., Gerbilliscus kempi, Taterillus gracilis) représentant $75 \%$ du total, ont été inventoriées. La prépondérance de Rattus rattus dans les captures avec 59,8\% de Mastomys sp. (13,5\%) et de C. olivieri (8,6\%) a été notée. Mus domesticus (0,29\%), Arvicanthis niloticus $(0,86 \%)$ et Crocidura sp. (0,86\%) sont faiblement représentées. L'indice de diversité de Shannon est de 2,08 dans la ville de Cotonou, de 2,70 dans les milieux non habités et de 1,45 dans les milieux habités où l'on ne trouve quasiment que les rats et souris. Le domaine de l'aéroport (aéroport international de Cotonou) et quelques zones marécageuses, inaccessibles à la population, constituent des zones refuges où l'indice de diversité des petits mammifères est relativement élevé.

() 2014 International Formulae Group. All rights reserved.

Mots clés: Inventaire, rongeurs, Cotonou, milieux non habités ou marginaux et habités.

\section{INTRODUCTION}

En 2000, $47 \%$ de la population mondiale habitaient dans les milieux urbains et en 2030, ce pourcentage passera à $60 \%$ (Baker et Harris, 2007). Ainsi, la population urbaine mondiale atteindra 2,1 milliards. Une telle explosion sera inévitablement favorable aux rongeurs commensaux, spécialement ceux dans les zones d'occupation informelle, sans système sanitaire, logements et infrastructures adéquats (Taylor et al., 2008). Cette situation augmentera le risque de la transmission des maladies zoonotiques. Malgré ce risque, les rongeurs urbains demeurent très peu étudiés du fait que les milieux urbains constituent un environnement inapproprié pour l'étude de l'écologie mammalienne (Taylor et al., 2008). Alors les travaux sur la diversité des rongeurs en milieux urbains sont rares, spécialement dans les villes africaines (Garba, 2012). 
La population urbaine augmente plus rapidement dans les pays en développement que dans les pays développés. Dans les villes africaines, la croissance démographique urbaine dépasse les capacités des gouvernements nationaux et locaux en matière de services de base. Elle suscite des problèmes de pauvreté difficiles à résoudre : chômage, logements insalubres, mauvaise qualité des systèmes d'assainissement.

Sous l'effet de cette pression démographique, l'urbanisation galope en l'absence d'une politique cohérente, respectueuse de la conservation de la biodiversité ou même d'un aménagement du territoire quelque peu rationnel. Cette urbanisation débridée, entraîne non seulement la destruction d'habitats intéressants pour la faune sauvage, mais aussi le remplacement de communautés animales autochtones par des généralistes opportunistes. Parmi eux, se trouvent des rongeurs tels que les rats (Rattus rattus et Rattus norvegicus) et les souris (Mus domesticus). Ainsi, les biocénoses urbaines de petits mammifères sont largement dominées par les espèces anthropophiles (Libois, 1984).

D'après Vieira et al. (2009), les petits mammifères (rongeurs et marsupiaux) sont spécialement utiles pour étudier les impacts des variables environnementales à cause de leur importance dans les systèmes naturels, aussi comme disséminateurs des graines, pollinisateurs, prédateurs des arthropodes, et aussi comme ressources alimentaires pour les carnivores, oiseaux de proie et reptiles. Si les petits mammifères, notamment les rongeurs, ont un rôle important dans les réseaux trophiques et dans la dynamique des écosystèmes, ils constituent aussi, en cas de pullulations, des agents de destruction des récoltes ou des produits stockés et peuvent s'avérer, en certaines circonstances, des facteurs de risque importants pour la santé humaine.

Les rongeurs au Bénin ont fait l'objet de nombreux travaux, particulièrement les espèces Thryonomys swinderianus Temminck, 1927, Cricetomys gambianus, Cricetomys emini (Codjia 1988, 1990 ; Codjia, 1995, 1998, 1999), les uns portant sur leur élevage en captivité, les autres sur leur écologie et leur dynamique dans les milieux naturels. Le plus récent, celui de De Visser et al. (2001), est un outil précieux pour l'identification des rongeurs du Bénin, mais aucun inventaire n'a été fait dans les milieux urbains et côtiers. Le présent travail est un complément d'informations sur l'écologie et la diversité des petits mammifères de la ville de Cotonou.

\section{MATERIEL ET METHODES Milieux d'études}

Deux groupes de milieux ont été distingués sur la base de la carte topographique de Cotonou (échelle $1 / 50.000^{\mathrm{e}}$ Feuille NB $n^{\circ}$ 31-XV- 1d): les milieux marginaux, MNH (non habités) et les zones plus proprement urbaines (MH) (Figure 1).

Les milieux marginaux sont caractérisés par la présence d'une couverture végétale importante. Il s'agit du domaine de l'Agence pour la Sécurité de la Navigation Aérienne (ASECNA, aéroport de Cotonou), de marécages herbacés et jardin.

a) Domaine de l'ASECNA.

Trois types d'habitats ont été retenus comme sites de piégeage sur la base des caractéristiques du sol et de la végétation: Poste A (domaine aéroport), Poste B (domaine aéroport) et Maboisé (domaine aéroport).

\section{- Poste A}

C'est un milieu sec au fourré littoral dégradé, fait de $60 \%$ de Poaceae (Heteropogon contortus (L.) Roem. \& Schult., Paspalum sp.) et de ligneux constitués de 20\% de Lauraceae (Cassytha filiformis L.), 15\% de Meliaceae (Azadirachta indica A. Juss), 4\% de Rutaceae (Zanthoxylum zanthoxylö̈des Lam.) et de $1 \%$ de Chrysobalanaceae (Chrysobalanus icaco L.), Celastraceae (Maytenus senegalensis (Lam.) Exell; Rhod.), Oleaceae (Jasminum dichotonum Vahl), Fabaceae (Indigofera hirsuta L.) et 
l'Amaranthaceae (Pandiaka involucrata (Moq). B.D. Jackson.

- $\quad$ Poste B (domaine aéroport PB)

Le sol est sablo-argileux avec une végétation de savane dominée à $90 \%$ par des espèces de Poaceae telles que Panicum maximum Jacq. et Pennisetum purpureum Schumach formant un tapis continu, Paspalum sp. réduit à un couloir et de Cyperaceae dont Killingaerecta Schumach. On y trouve des espèces ligneuses telles que Vitex donania Sweet., Elaeis guineensis Jacq., Cassytha filiformis L, Triumfetta rhomboidea Jacq. (Tiliaceae). On note la présence d'une mare à une dizaine de mètres des lieux.

- $\quad$ Maboisé (domaine aéroport)

C'est un marécage boisé constitué par le bourrelet de la mare et sa berge. Le bourrelet porte la végétation de Nephrolepis sp., de Ficus capensis Thunb comme ligneux en peuplement assez fourni. Il est situé à l'ouest d'une mare jonchée de Typha australis Schumach et de Nephrolepisbisserta. Dans le couvert végétal de la mare, il y a une petite Cyperus sp.

b) Marécage herbacé

C'est une savane marécageuse sur un sol hydromorphe, composée à 99\% de Typha australis Schumach (Typhaceae). Parmi les autres espèces relevées, notons Ipomeaaquatica Forsst. (Convolvulaceae) et Aneilimabeniniense (P. Beauv) Kunth. (Commelinaceae).

c) Jardins

Il s'agit du domaine annexe de l'aéroport érigé en zone de cultures par quelques maraîchers. On y cultive la carotte (Daucus carota L.) (Opiaceae), les choux (Brassica sp. Brassicaceae), le concombre (Cucumissativus L.) Cucurbitaceae) et les épinards (Spinaciaoleracea L.) (Amaranthaceae)

Les milieux habités, MH (les quartiers) sont caractérisés par la nature du sol, la présence ou l'absence de caniveaux à ciel ouvert, la présence éventuelle de dépotoirs sauvages et, l'existence possible de structures de collecte des déchets. Il s'agit des quartiers de Godomey et Dantokpa du côté Ouest du chenal reliant le lac Nokoué à l'océan et d'Abokicodji et de Tchankpamin du côté Est. Dantokpa et Abokicodji sont situés en vis à vis. Dantokpa est construit sur un sol sabloargileux et abrite un grand marché, de caniveaux à ciel ouvert et de structures de collecte des déchets. Abokicodji est sur un sol sableux, sans caniveau à ciel ouvert. Tchankpamin et Godomey sont distants d'au moins $15 \mathrm{~km}$ l'un de l'autre. Godomey est un milieu sec disposant d'importants dépotoirs. Tchankpamin est construit sur un sol humide inondable en saison pluvieuse. Les ordures sont parfois utilisées par certaines personnes pour remblayer leurs parcelles marécageuses. À ces quatre quartiers principaux s'ajoutent des quartiers secondaires. Il s'agit, du côté ouest du chenal, du Restaurant universitaire, des quartiers de Ste Rita, Aîbatin, Houeyiho, St Michel, Missèbo et du côté est, d'AkpakpaDodomey, Sodjatinmey, Suru-Lere, Avotrou, Midombo, Sènadé.

\section{Campagnes de piégeage}

Les campagnes de piégeage se sont déroulées du 25 août 2004 au 25 mai 2005 avec interruptions. Quatre-vingt pièges dortoirs grillagés (fabrication artisanale inspirée du modèle Firobin) et plusieurs types d'appât (poisson fumé, farine de blé pétrie avec des sardines à l'huile, noix de palme) ont été utilisés. Dans les milieux urbanisés, les captures ont été effectuées chaque mois. Une dizaine de pièges ont été placés à l'intérieur des chambres ou des cuisines. Dans chaque milieu périphérique, une campagne unique a été effectuée pour recenser les espèces. Une vingtaine de pièges ont été placés à intervalles de $5 \mathrm{~m}$. Les pièges, une fois installés, sont relevés chaque matin pendant trois jours soit trois nuits par piège. Les pièges ayant capturé des petits mammifères sont remplacés. Les animaux capturés ont été transportés au laboratoire où ils ont été anesthésiés avec de l'éther, puis pesés à l'aide d'un peson à ressort 
précis au gramme près, sexés et identifiés à l'espèce suivant l'ouvrage de De Visser et al. (2001). Les coordonnées de chaque animal sont inscrites avec un numéro d'ordre dans le répertoire.

\section{Etude de la diversité des petits mammifères}

Plusieurs indices ont été calculés. Il s'agit de:

i) l'indice d'abondance (éventuellement spécifique): nombre de captures par effort de piégeage (nombre de pièges-nuits);

ii) l'indice de diversité spécifique de Shannon (H'): $\mathrm{H}^{\prime}=-\Sigma \mathrm{P}_{\mathrm{i}} \log _{2}\left(\mathrm{P}_{\mathrm{i}}\right)$ avec $\mathrm{P}_{\mathrm{i}}: \mathrm{N}_{\mathrm{i}} / \mathrm{N}$, $\mathrm{N}_{\mathrm{i}}=$ effectif de l'espèce $\mathrm{i}, \mathrm{N}=$ effectif total et i;

iii) l'indice de régularité de Piélou (E): $\mathrm{E}=\mathrm{H}^{\prime} / \log _{2} \mathrm{~S}$ avec $\mathrm{S}=$ nombre total d'espèces.

Ces indices permettent de mieux rendre compte de l'organisation fonctionnelle et de l'évolution de la communauté (Daget, 1976; Barbault, 1992).

Le test de l'écart-réduit a été utilisé pour analyser d'éventuelles différences entre le sex-ratio observé et le sex-ratio théorique 1:1. La comparaison de deux proportions a été réalisée grâce au test bilatéral de $\mathrm{Z}$.

\section{RESULTATS}

\section{Espèces capturées}

A la fin de nos campagnes, nous avons consenti un effort de piégeage de 2652 nuitspièges réparties dans 21 stations. Au total, 348 petits mammifères ont été capturés et répartis sur 11 espèces appartenant à 2 familles. La plus représentée a été celle des Muridae avec 9 espèces. Les Soricidae ont été représentées par 2 espèces. Trois espèces, Rattus rattus, Rattus norvegicus et Mus domesticus, ont été cosmopolites. Les huit autres, Crocidura olivieri, Crocidura sp, Arvicanthis niloticus, Cricetomys gambianus, Dasymys rufulus, Mastomys sp, Gerbilliscus kempi, Taterillus gracilis, ont été autochtones.

\section{Abondance des espèces}

L'espèce Rattus rattus a été la plus prépondérante avec $59,8 \%$ suivie de Mastomys sp. (13,5\%) et de C. olivieri (8,6\%). Les espèces Mus domesticus (0,3\%), Arvicanthis niloticus $(0,9 \%)$ et Crocidura sp. $(0,9 \%)$ ont été très faiblement représentées.

L'indice d'abondance des captures par milieu est présenté dans le Tableau 1. L'indice d'abondance global (espèces et stations confondues) a été de 0,110 dans le milieu non habité (MNH) et de 0,133 dans le milieu habité (MH). La moyenne des indices d'abondance a été de 0,010 $\pm 0,011$ dans le MNH et de 0,011 $\pm 0,027$ dans le MH. Dans le $\mathrm{MH}$, l'indice d'abondance le plus élevé $(0,093)$ a été observé chez Rattus rattus tandis que le plus faible est observé chez Arvicanthis niloticus et Mus domesticus. Certaines espèces ont été présentes dans un seul milieu. Les espèces Crocidura sp., Dasymys rufulus, Taterillus gracilis ont été présentes dans le MNH et Rattus norvegicus, Mus domesticus et Cricetomys gambianus dans le MH. Les espèces Crocidura olivieri, Arvicanthis niloticus et Gerbilliscus kempi ont été plus abondantes dans les MNH alors que Mastomys sp. et surtout Rattus rattus ont été intimement associés aux MH.

La répartition des individus par sexe et par espèce pour les espèces les plus représentées (nombre d'individus $\geq 10$ ) a été présentée dans le Tableau 2. Le sex-ratio ne s'est pas écarté significativement du rapport théorique de $1: 1$ sauf chez $R$. norvegicus où le nombre de mâles capturés a été en excès par rapport à celui des femelles (Ecart réduit $=0,96$ N.S.).

Les indices d'abondance mensuels sur les deux ans ont été présentés à la Figure 2. L'indice présente une chute en février 2004 et 2005 qui s'est accentuée en mars 2005. En 2004, il a montré un sommet qui s'est étalé d'avril à juillet. En revanche, en 2005, un pic assez net en mai a été observé. Cependant, la différence entre les indices d'une année à l'autre pour le même mois, testée au moyen de 
Z bilatéral, n'a pas été significative pour février et avril, mais significative pour janvier, mars (limite) et mai (Tableau 3).

\section{Diversité spécifique des petits mammifères}

Le Tableau 4 a présenté les indices de diversité de Shannon et d'équitabilité de Piélou par milieu et par station. L'indice de diversité dans la ville de Cotonou a été de 2,08 avec un indice d'équitabilité égal à 0,58.
Dans le MNH, l'indice de diversité a été de 2,70 donc supérieur à 1,45 , celui du $\mathrm{MH}$.

Pour les stations: Abokicoji, Aïbatin, Akpakpa Dodomey, Maboisé, Sènadé, Sodjatinmin, St Michel, St Rita, SuruLere, les indices n'ont pas été calculés, l'effectif des individus capturés étant faible $(<10)$. Dans les stations retenues, l'indice le plus élevé a été enregistré à Missèbo et le plus faible au Jardin.

Tableau 1: Indices d'abondance par milieu.

\begin{tabular}{lcccc}
\hline & Milieu non habité (432 pièges nuits) & Milieu habité (2220 pièges nuits \\
\hline Espèces & Nb captures & Indice d'abondance & Nb captures & Indice d'abondance \\
\hline Arvicanthis niloticus & 2 & 0,004 & 1 & 0 \\
Cricetomys gambianus & 0 & 0 & 4 & 0,002 \\
Crocidura olivieri & 11 & 0,022 & 19 & 0,009 \\
Crocidura sp. & 3 & 0,006 & 0 & 0 \\
Dasymys rufulus & 10 & 0,02 & 0 & 0 \\
Mus domesticus & 0 & 0 & 1 & 0 \\
Mastomys sp. & 1 & 0,002 & 46 & 0,021 \\
Rattus norvegicus & 0 & 0 & 207 & 0,007 \\
Rattus rattus & 1 & 0,002 & 0 & 0,096 \\
Taterillus gracilis & 13 & 0,3 & 2 & 0 \\
Gerbilliscus kempi & 11 & 0,022 & 296 & 0,001 \\
Total & 52 & 0,106 & & 0,137 \\
\hline
\end{tabular}

Tableau 2: Sex-ratio des captures par espèce.

\begin{tabular}{lcccc}
\hline Espèce & Femelles & Mâles & Total & Écart réduit \\
\hline Arvicanthis niloticus & 1 & 2 & 3 & \\
Cricetomys gambianus & 2 & 2 & 4 & \\
Crocidura olivieri & 20 & 10 & 30 & 1,83 N.S. \\
Crocidura sp. & 0 & 3 & 3 & \\
Dasymys rufulus & 6 & 4 & 10 & 0,63 N.S. \\
Mus domesticus & 1 & 0 & 1 & \\
Mastomys sp. & 26 & 21 & 47 & 0,73 N.S. \\
Rattus norvegicus & 3 & 13 & 16 & 2,5 (p $<05$ ) \\
Rattus rattus & 112 & 96 & 208 & 1,11 N.S. \\
Taterillus gracilis & 6 & 7 & 11 & 0,9 N.S. \\
Gerbilliscus kempi & 8 & 5 & 13 & 0,83 N.S. \\
Total & 183 & 165 & 348 & 0,96 N.S.
\end{tabular}


Tableau 3: Différence entre les captures de 2004 et 2005 pour le même mois (test bilatéral de Z).

\begin{tabular}{lccc}
\hline Mois & Captures & Pièges posés & Test \\
\hline janvier-04 & 26 & 260 & $\mathrm{Z}=2,37$ \\
janvier-05 & 24 & 120 & $\mathrm{p}=0,018$ \\
février-04 & 18 & 260 & $\mathrm{Z}=0,278$ \\
février-05 & 10 & 120 & $\mathrm{p}=0,781$ \\
mars-04 & 30 & 245 & $\mathrm{Z}=1,965$ \\
mars-05 & 8 & 135 & $\mathrm{p}=0,049$ \\
avril-04 & 32 & 60 & $\mathrm{Z}=1,099$ \\
avril-05 & 13 & 501 & $\mathrm{p}=0,272$ \\
mai-04 & 73 & 60 & $\mathrm{Z}=2,099$ \\
mai-05 & 15 & $\mathrm{p}=0,036$ \\
\hline
\end{tabular}

Tableau 4: Indices de diversité spécifique par station et par milieux.

\begin{tabular}{lcccc}
\hline Stations & Nb espèces & Nbre individus & $\mathbf{H}^{\prime}$ & $\mathbf{E}$ \\
\hline Global & 12 & 348 & 2,08 & 0,58 \\
Milieux non habités & 9 & 40 & 2,7 & 0,85 \\
Milieux habités & 8 & 308 & 1,45 & 0,48 \\
Abokicoj & 1 & 7 & - & \\
Aïbatin & 1 & 3 & - & - \\
AkDodomey & 2 & 2 & - & - \\
ASEC PA & 2 & 13 & 0,619 & 0,619 \\
ASEC PB & 3 & 14 & 0,946 & 0,597 \\
Avotrou & 5 & 25 & 1,539 & 0,663 \\
Dantokpa & 4 & 45 & 1,669 & 0,835 \\
Gbadji & 4 & 18 & 1,098 & 0,549 \\
Godomey & 4 & 63 & 0,929 & 0,465 \\
Jardin & 2 & 12 & 0,414 & 0,414 \\
Maboisé & 2 & 3 & - & - \\
Maher & 2 & 10 & 0,722 & 0,722 \\
Midombo & 5 & 41 & 1,226 & 0,528 \\
Missèbo & 4 & 11 & 1,823 & 0,912 \\
Restau U & 2 & 19 & 0,485 & 0,485 \\
Sènadé & 3 & 9 & - & - \\
Sodjatinmin & 3 & 4 & - & - \\
St Michel & 2 & 9 & - & - \\
St Rita & 2 & 6 & - & - \\
SuruLere & 4 & 8 & - & - \\
& & & & \\
Tchankpa & 3 & 26 & 0,929 & 0,586 \\
\hline & & & & \\
\hline
\end{tabular}




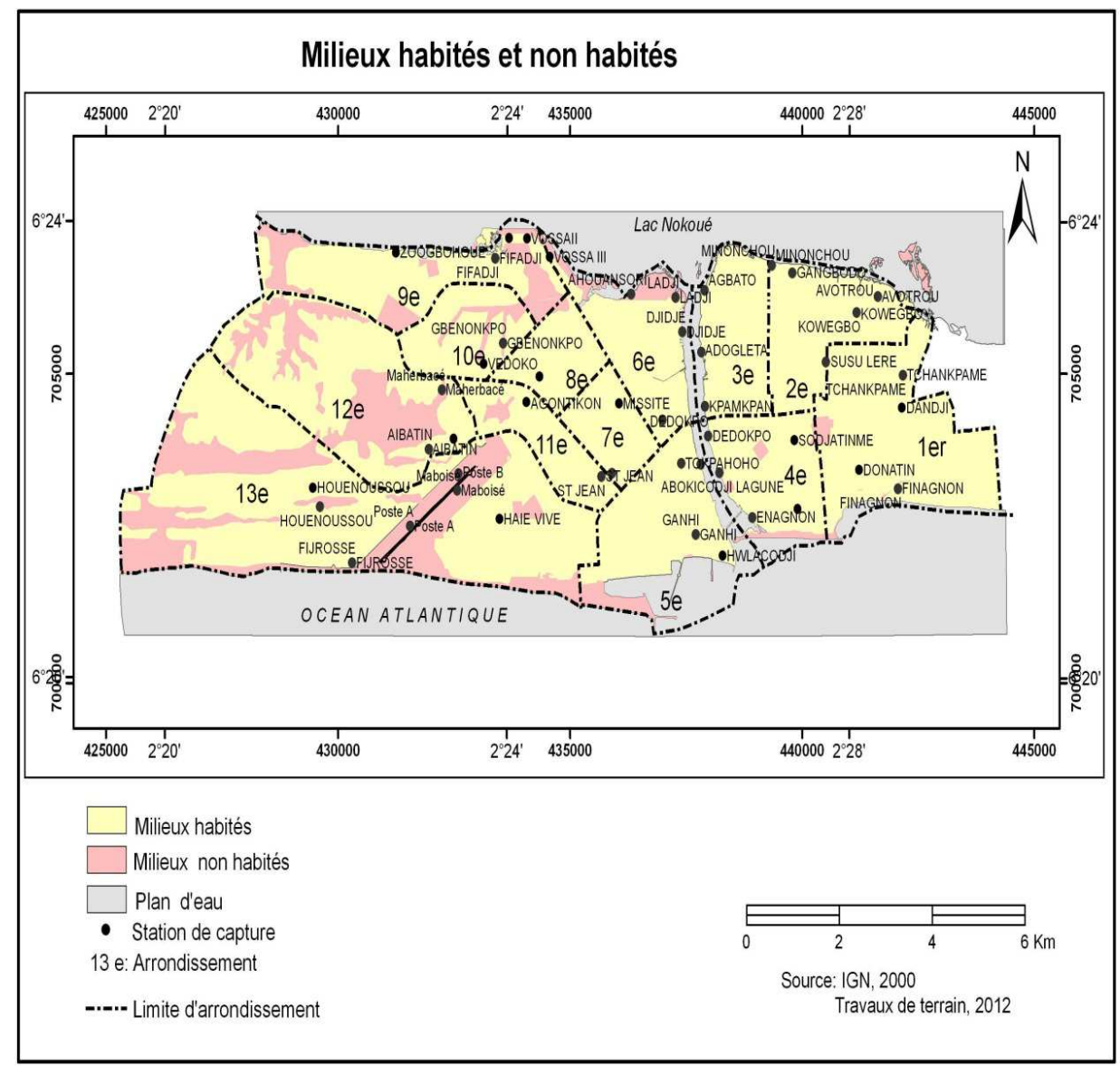

Figure 1 : Répartition des stations de capture dans les milieux habités et non habités.

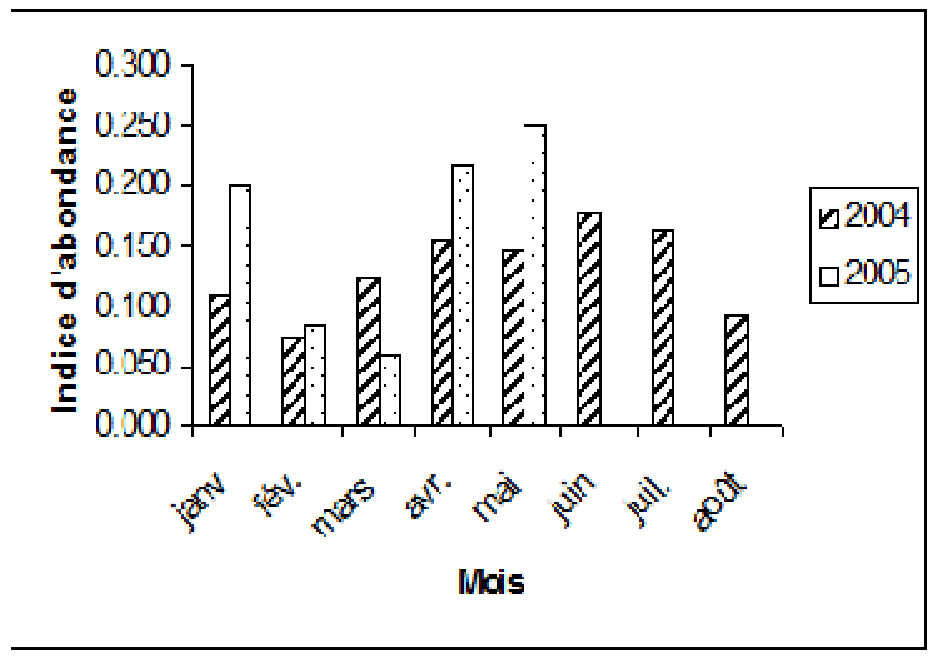

Figure 2: Evolution mensuelle des indices d'abondance globale des micromammifères. 


\section{DISCUSSION}

Les espèces de Muridae sont prépondérantes dans nos captures $82 \%(9 / 11)$. Ils sont plus abondants que les insectivores $18 \%(2 / 11)$. Ce résultat confirme le point de vue de Wilson et Reeder (1993) et Huchon et al. (2002) selon lequel les rongeurs sont les plus abondants et le plus diversifiés. L'indice d'abondance global des petits mammifères de la ville de Cotonou est de 0,131. Il est supérieur à celui de Soliman et al. (2001) en milieu rural en Egypte (0,086), de Bakr (1996) à Shebin toujours en Egypte $(0,05)$ et sensiblement identique à celui de Laudisoit (2004) à Kinshasa (0,133). L'indice d'abondance global des petits mammifères, bien qu'il soit relativement élevé, nous pensons qu'il demeure faible du fait de la forte présence de prédateurs, des rongeurs tels que le chat en occurrence. En effet, nombre de béninois prennent des chats dans leur maison non pas pour leur compagnie, mais surtout pour éloigner rats et serpents des habitations. Nous pouvons également évoquer l'inefficacité de certains pièges liée à la dimension trop grande des mailles du grillage employé et qui ne permet pas d'attraper les espèces de petite taille comme les souris (Mussp.).

Les individus de Rattus rattus ont réalisé l'indice d'abondance le plus élevé $(0,23)$ à Gbadji et au Restaurant universitaire. La station de Gbadji est faite d'environ une centaine de logements implantés dans un milieu marécageux, pour la plupart de fortune où règne une véritable insalubrité (immondices et eaux stagnantes) attirant les rats, ce qui justifie un tel résultat. Restaurant universitaire, les restes alimentaires mal stockés attirent les rats la nuit.

Dans la ville de Cotonou, 11 espèces de petits mammifères se partagent 21 stations regroupées en deux types de milieux sur la base des variables "milieu" et des variables "espèces" qui leurs sont propres. Cette répartition est liée à leurs régimes alimentaires et à leur affinité pour une niche écologique particulière (Codjia, 1995; Daouda, 2002). Il s'agit du:

- MNH ou milieu marginal colonisé par les espèces forestières, Gerbilliscus kempi, Taterillus gracilis, Arvicanthis niloticus, Crocidura sp., ayant un régime alimentaire particulier composé de racines, de tubercules, de fruits, de feuilles et d'insectes. Il est caractérisé par la présence d'arbres, d'une strate herbacée développée, l'absence d'implantations humaines et d'ordures ménagères.

- MH ou habitations humaines qui regroupe 17 stations et abrite en majorité des espèces commensales, Rattus rattus, Rattus norvegicus, Mastomys sp. à régime omnivore. La présence de Gerbilliscus kempi dans ce milieu, précisément à Avotrou et Midombo, s'explique par l'existence de formations herbeuses derrière certaines habitations dans ces stations. L'indice de diversité de Shannon est de 2,08 pour toute la ville de Cotonou, de 2,70 pour le $\mathrm{MNH}$ et de 1,45 pour le MH. On observe que le MNH abrite la communauté la plus diversifiée. La majorité des espèces y est présente avec un indice d'abondance variant de 0,020 à 0,22 chez Dasymys rufulus Taterillus gracilis, Tatera kempi et Crocidura olivieri. Ce milieu comprend en grande partie le domaine de l'ASECNA qui dispose de plusieurs types d'habitats avec une couverture végétale variée. Les individus de 9 des 11 espèces y sont capturés et ceux de 3 espèces lui sont propres. Il s'agit de Crocidura sp. dans le marécage boisé, de Dasymys rufulus dans la savane marécageuse à Typha sp. et de Taterillus gracilis dans le milieu sabloargileux en bordure de la mare. Ces résultats sont conformes à ceux de Liat et al. (1980) qui, étudiant les petits mammifères et leurs ectoparasites dans divers habitats à l'ouest de Java, ont trouvé que c'est dans les milieux les plus diversifiés en habitats qu'on trouve la 
diversité la plus élevée de petits mammifères. L'indice de diversité faible dans le MH peut s'expliquer par l'abondance de l'espèce Rattus rattus. Dans la ville de Cotonou, l'espèce $R$. rattus est la plus largemnt répandue et plus abondante (Houémènou et al., 2013).

L'espèce Cricetomys gambianus se trouve dans tout le Bénin tant dans les milieux secs que dans les milieux humides (De Visser et al., 2001). Son absence dans nos captures dans le MNH est surprenante étant donné que ce milieu contient divers habitats et des espèces végétales comme Elaeis guineensis affectionne les noix. Nous pourrions expliquer cette absence par la présence de ses prédateurs naturels mais surtout par la taille de nos pièges relativement petite qui ne permet pas sa capture.

Dans les stations, l'indice de diversité le plus faible est observé dans les jardins $(0,414)$ tandis que le plus élevé est observé à Missèbo $(1,823)$.

L'indice d'abondance global est de 0,131 (348 rats/2652 nuits-pièges). Il est plus élevé dans le MH $(0,137)$ que dans le MNH $(0,106)$. Dans 14 stations, il est $\geq 0,10$. La station de Gbadji présente l'indice d'abondance le plus élevé $(0,30)$. La disponibilité des ressources alimentaires pour les petits mammifères et la présence des herbes autour des habitations pourraient justifier un tel résultat.

Les individus de Mastomys sp. sont présents dans 7 stations sur 21 avec des indices d'abondance presque identiques $(0,02$ à 0,03 ). Sa capture a été réalisée dans les stations telles que Abokicodji, Gbadji, Godomey, Ste Rita, SuruLere, Tchankpa où on observe aux alentours de certaines habitations de la végétation. Son absence à la station de Dantokpa et de Missèbo, grands marchés au centre de la ville, confirme les résultats de De Visser et al., 2001 qui ont affirmé que c'est une espèce qui vit dans les maisons. Mais ces auteurs n'ont pas intégré dans leur analyse la présence d'un tapis herbacé aux alentours de ces habitations.

Les individus de Crocidura olivieri sont retrouvés dans 9 stations sur 21 avec des valeurs d'indice d'abondance allant de 0,01 à 0,18 . C'est une espèce ubiquiste dans la ville de Cotonou. Sa rareté dans certaines stations s'explique par le fait que la plupart des pièges ont été posés à l'intérieur des chambres où, contrairement à Rattus rattus, Crocidura olivieri n'a pas accès.

De façon globale, les individus de trois espèces sont les plus abondants dans nos captures: deux Muridés, Rattus rattus $(59,77 \%)$ et Mastomys sp. (13,5\%) et un Soricidé Crocidura olivieri 8, 6\%. La capture d'un grand nombre de Rattus rattus est liée à sa grande distribution dans le monde (Vanasco et al., 2003; Wilson et Reeder, 2005) et sa présence attestée dans d'autres villes africaines comme Alexandrie et Caire, Egypte (Morsy et al., 1988; Khalid et al., 1992), Kinshasa, RDC (Laudsoit, 2004), Makurdi, Nigéria (Omudu et Ati, 2010), Hararé, Zimbabwé ou Niamey, Niger (Garba, 2012). Le nombre relativement élevé de Mastomys sp. et Crocidura olivieri est soutenu par de récentes études en Afrique (RatZooMan, 2012). Ces résultats se rapprochent de ceux de Bakret al. (1996) qui, étudiant les puces infestant les petits mammifères de la ville de Shebin en Egypte, ont noté l'abondance de Rattus rattus (45\%) suivie de celle de Rattus norvegicus (30,5\%) et de Mus musculus (24,2\%). Ils sont différents de ceux de Soliman et al. (2001) et de Laudisoit (2004) par les espèces en cause et leur abondance relative. Chez les premiers, deux espèces ont été capturées dans 3 stations d'étude contre 11 sur 21 pour notre étude. Rattus norvegicus est abondant à 53,4\% tandis que dans nos captures c'est Rattus rattus qui domine à 59,77\%.

A Cotonou, Rattus norvegicus ne représente que $4,5 \%$ contre $59,77 \%$ pour 
Rattus rattus. Le rat noir $R$. rattus semble plus adapté aux conditions de l'Afrique comparativement au rat brun Rattus norvegicus (Gratz et Arata; 1975). Rattus norvegicus est capturé dans 3 stations et réalise l'abondance la plus élevée à Dantokpa, l'immense marché de Cotonou. Cette abondance relativement élevée (3\%), reste faible car les rats de cette station sont trop gros et n'arrivent pas à entrer dans nos pièges.

La répartition des espèces n'est pas influencée par la barrière naturelle que devait constituer pour elles, la lagune de Cotonou. Ainsi, en dehors des espèces forestières qui sont piégées dans les MNH situés à l'ouest de la lagune, les espèces commensales les plus abondantes sont présentes dans les stations situées de parts et d'autres de la lagune. On retrouve les espèces telles que Rattus rattus, Mastomys sp., Crocidura olivieri dans les stations situées à l'est de la lagune comme Abokicoji, Midombo, à l'ouest, Dantokpa et Midombo. Cette égale répartition peut se justifier d'une part, par l'existence des ponts qui relient les deux côtés de la lagune et que les petits mammifères peuvent passer pour aller d'une station à l'autre, d'autre part, ils peuvent profiter des embarcations que les commerçants utilisent pour transporter leurs marchandises au marché Dantokpa.

\section{Conclusion}

Deux groupes de milieux, les milieux «marginaux» ou non habités et les milieux habités «les quartiers» se partagent les petits mammifères de la ville de Cotonou. Les premiers hébergent les espèces forestières et les deuxièmes, les espèces anthropophiles. La communauté de petits mammifères de la ville de Cotonou est riche de 11 espèces et est dominée par Rattus rattus. Le domaine de l'ASECNA et quelques zones profondément marécageuses inaccessibles à la population, milieux non habités ou marginaux, constituent des zones refuges où l'indice de diversité des petits mammifères est le plus élevé.

\section{REMERCIEMENTS}

Nous sommes reconnaissants envers l'Etat béninois pour avoir financé nos études à l'Université de Liège en Belgique. Nous remercions toutes les personnes qui ont aidé à la réalisation de cette étude, particulièrement: Pierrette de SOUZA et Docteur Alphonse CHABI, pour la collecte des données et Houéto Félix pour la réalisation de la carte.

\section{RÉFÉRENCES}

Baker JP, Harris S. 2007. Urban mammals: what does the future hold? An analysis of the factors affecting patterns of use of residential gardens in Great Britain. Mam. Rev., 37(4) 297-315.

Bakr EM, Morsy AT, Nassef AEN, El Meligi AM. 1996. Fleas ectoparasites of commensal rodents in ShebinEl Kom, Menoufia Governorate, Egypte. J. Egypt. Soc. Parasitol., 26(1): 39-52.

Barbault R. 1992. Écologie des Peuplements: Structure, Dynamique et Evolution. Masson.

Codjia JTC. 1988. Problématique liée à l'utilisation du gibier et écoéthologie de quelques rongeurs consommés au Bénin. Nature et Faune, 4(4): 4-16.

Codjia JTC. 1990. Elevage expérimental des cricétomes (Cricetomys gambianus et $C$. emini). Note d'informations. Description de quelques comportements. Nature et Faune, 6(1): 35-44.

Codjia JTC. 1995. Répartition écologique et dynamique des populations de cricétomes (Cricetomys gambianus et Cricetomys emini) de l'aulacode (Thryonomys swinderianus) dans les milieux naturels du Bénin. Thèse Doctorat Sciences Zoologiques, $\mathrm{PhD}$, Université de Liège, Belgique, P 210. 
Codjia JTC. 1998. Rôle écologique, économique et sociculturel des rongeurs. Communication. In Actes des journées de réflexion du ReRE sur les rongeurs et leurs prédateurs au Bénin, p11.

Codjia JTC. 1999. Facteurs explicatifs de la répartition écologique des rongeurs du Bénin: cas des cricétomes (Cricetomys gambianus et Cricetomys emini). In Actes séminaire National Rongeurs Ophidiens. Cotonou 24-28 mars 1997. Edition Flamboyant. Bénin.

Daget J. 1976. Les Modèles Mathématiques en Ecologie. Eds. Masson: Paris; 172 p.

Daouda AHI. 2002. Caractéristiques staturopondérales, parasitisme par les cestodes et dynamique des populations chez les rongeurs de la presqu'île du Cap-Vert (Sénégal). Thèse de Doctorat de $3^{\mathrm{e}}$ cycle. Université Cheikh Anta Diop de Dakar. $143 \mathrm{p}$.

De Visser J, Mensh GA, Codjia JTC, Bokonon-Gantha HA. 2001. Guide préliminaire de reconnaissance des rongeurs du Bénin. Réseau Rongeurs et Environnement. CBDD.eC Cooperation. Bénin, $252 \mathrm{p}$.

Firdaus G. 2012. Urbanization, emerging slums and increasing health problems: a challenge before the nation: an empirical study with reference to state of Uttar Pradesh in India. J. Environ. Res. Managem., 9: 146-152.

Garba M. 2012. Rongeurs urbains et invasion biologique dans le sud-ouest du Niger : écologie des communautés et génétique des populations. Thèse de doctorat. Université Abdou Moumouni de Niamey. Niger, 261 p.

Gratz NG, Arata AA. 1975. Problems associated with the control of rodents in tropical Africa. Bull. W.H.O., 52(4-6): 697-706.

Huchon D, Madsen O, Sibbald MJJB, Ament K, Stanhope MJ, Catzeflis F, De Jong
WW Douzery EJP. 2002. Rodent phylogeny and a timescale for the evolution of glires: evidence from an extensive taxon sampling using three nuclear genes. Mol. Biol., 19: 1053-1065.

Khalid LM, Morsy TA, el Shennawy SF, Farrag AM, Sabry AH, Mostafa HA. 1992. Studies on flea fauna in El Fayoum Governorate, Egypte. J. Egypt. Soc. Parasitol., 3: 783-799.

Laudisoit A. 2004. Les ectoparasites des petits mammifères de la ville de Kinshasa, République Démocratique du Congo : un facteur de risques pour la santé publique ? Mémoire de DEA en Biologie Animale. Unité Recherches Zoogéographiques, Université de Liège, p.75.

Liat LB, Sustriayu N, Hadi RT, Bang HY. 1980. A study of small mammals in the Ciloto fields station area, west Java, Indonesia, with special reference to vectors of plague and scrub typhus. Southeast As. J. Trop. Med. Pub. Health, 11(1): 71-80.

Libois MR. 1984. Le régime alimentaire de la Chouette effraie. Essai synécologique sur les micromammifères d'Europe et ouest méditerranéen. Etude par l'analyse du régime alimentaire de la Chouette effraie, Tyto alba (SCOPOLI). Thèse de Doctorat. Université de Liège, 184 p.

Morsy TA, Abou El-Ela RG, El Gozamy BMR. 1988. The commensal rodents and their flea fauna in Alexandria City, Egypt. J. Egypt. Soc. Parasitol., 18: 11-28.

Omudu EA, Ati TT. 2010. A survey of rats trapped in residential apartments and their ectoparasites in Makurdi, Nigeria. Res. J. Agric. Biol. Sci., 2: 144-149.

Patergnani M, Gras LM, Poglayen G, Gelli A, Pasqualucci F, Farina M, Stancampiano L. 2010. Environmental influence on urban rodent bait consumption. J. Pest. Sci., 83(3): 347-359. 
RatZooMan. 2012. http://www.nri.org/ projects/ratzooman/publications.htm

Soliman S, Marzouk AS, Main AJ, Montasser AA. 2001. Effect of sex, size, and age of commensal rat hosts on the infestation parameters of their ectoparasites in a rural area of Egypt. J. Parasitol., 87(6): 13081316.

Taylor JP, Arntzen L, Hayter M, Iles M, Frean J, Belmain S. 2008. Understanding and managing sanitary risks due to rodent zoonoses in an african city: beyond the Boston Model. Integr. Zool., 3: 38-50.

Vanasco NB, Sequeira MD, Sequeira G, Tarabla HD, 2003. Associations between leptospiral infection and seropositivity in rodents and environmental characteristics in Argentina. Prevent. Vet. Med., 60: 227235.

Vieira VM, Olifiers N, Delciellos CA, Antunes ZV, Bernado RL, Grelle VEC, Cerqueira R. 2009. Land use vs, fragment size and isolation as determinants of small mammal composition and richness in Atlantic Forest remnants. Biol. Conserv., 142: 1191-1100.

Wilson DE, Reeder DM. 1993. Mammal Species of the World. SmithsonianInstitution Press: Washington, D.C.; 1207. 